\title{
Manifestation der angeborenen Zwerchfellhernie und molekulare Grundlagen
}

\author{
Manifestation of a Congenital Diaphragmatic Hernia and Molecular Pathogenesis
}

L. Gortner

Bibliografie

DOI http://dx.doi.org/

$10.1055 / \mathrm{s}-0032-1329994$

Klin Padiatr 2012; 224: 429-430

(c) Georg Thieme Verlag KG

Stuttgart · New York

ISSN 0300-8630

Korrespondenzadresse

Prof. Dr. Ludwig Gortner

Klinik für Allgemeine Pädiatrie

und Neonatologie

Kliniken für Kinder- und Jugend-

medizin

Universitätsklinikum des

Saarlandes

Gebäude 9

66421 Homburg/Saar

ludwig.gortner@uks.eu
Die angeborene Zwerchfellhernie ist ein neonataler Notfall mit immer noch substantieller Mortalität [3]. Sie ist die häufigste Form der Lungenfehlbildungen sekundärer Art, welche durch eine Verdrängung der Lunge durch das Eventerat und damit einer Wachstumsstörung bedingt ist, und wird bei 1:2000-1:4000 aller Neugeborenen beobachtet. Die Lokalisation ist mit rund 8:1 überwiegend linksseitig. Eine pränatale Diagnostik dieser Fehlbildung ist absolut dringlich und notwendig, da dies zu einer nennenswerten Verbesserung der Prognose durch nachstehend aufgeführte Maßnahmen führte [5]:

1. Durch eine Geburt in einem Zentrum der Maximalversorgung kann eine sachgerechte postnatale neonatologische intensivmedizinische Therapie umgehend eingeleitet werden. Dies bedeutet, dass ein für die betroffenen Neugeborenen gefährliches und damit obsoletes Blähmanöver der Lunge unterbleibt, was seinerseits die Lungenfunktion durch zunehmende Luftfüllung der eventerierten Abdominalorgane weiter einschränkt. Eine klinische Diagnose ist in der gegebenen Notfallsituation schwierig und findet in Leitlinien keine Erwähnung [15].

2. Weiterhin wurden in der diagnostischen Ebene bedeutsame Fortschritte durch die in Ergänzung zur pränatalen sonografischen Diagnostik eingesetzten MRT-Untersuchungen intrauterin gemacht, welche mittels Bestimmung der Lungenvolumina eine recht zuverlässige prädiktive Abschätzung der Notwendigkeit einer postnatalen, meist postoperativen Behandlung mittels einer extrakorporalen Membranoxygenierung (ECMO) möglich machen [12]. Somit ist eine adäquate Allokation der Betroffenen innerhalb von Zentren mit ECMOAusstattung pränatal möglich.

3. Die pränatale Therapie der Zwerchfellhernie nach entsprechender Diagnostik wurde in den 80er Jahren durch die Gruppe von Adzick und Harrison [1] inauguriert, wobei sich der Ansatz des pränatalen Zwerchfellverschlusses nicht durchsetzte, da der Eingriff per se ein hohes Risiko beinhaltete. Ein neuerer seit rund 10 Jahren verfolgter Ansatz ist die pränatale Applikation einer trachealen Okklusion durch fetale Endoskopie als eine minimalinvasive Prozedur, was in kleineren Fallserien mit einer verbesserten Überlebensrate bei prognostisch ungünstig kategorisierten Verläufen einherging. Eine große internationale Studie zur Evaluation dieses Ansatzes mittels trachealer Okklusion (tracheal occlusion to accelerate lung growth trial - TOTAL-trial) ist auf dem Weg, um den Stellenwert dieser pränatalen Therapie zu evaluieren $[6,8]$. Unter dem Aspekt der Lungenphysiologie ist jede Behandlung sinnvoll, die der Lunge pränatal während kritischer Phasen der terminalsacculären und alveolären Periode ein regelrechtes oder nur wenig eingeschränktes Wachstum ermöglicht, um so die gefürchtete Hypoplasie der Lunge auf der betroffenen Seite zu verhindern bzw. zu attenuieren.

Die 3 zuvor genannten Punkte belegen, dass eine adäquate pränatale Diagnostik betroffener Neugeborener dringlich ist und dies wie zu erwarten zu einer insgesamt verbesserten Prognose durch gezielten Einsatz einer adäquaten intensivmedizinischen Betreuung geführt hat. Diese Möglichkeiten bestehen bei einer späten Manifestation von Zwerchfellhernien nicht. Es wird allgemein davon ausgegangen, dass das Eventerat in der pränatalen Phase nicht darstellbar ist und somit ist die Möglichkeit der pränatalen Diagnostik nicht gegeben. Auch ist die Möglichkeit der klinischen Diagnosestellung im Rahmen der frühen Vorsorgeuntersuchungen U 1 bis U 3 aus zuvor genannten Gründen nicht gegeben.

Daher muss bei allen unklaren Zuständen einer respiratorischen Insuffizienz ohne erkennbare infektiöse oder kardiale Grunderkrankung an die Entität der späten Zwerchfellhernie gedacht werden. Hierbei variiert, wie die hier in der Klinischen Pädiatrie $[2,13]$ und anderenorts geschilderten Verläufe belegen, die klinische Präsentation von langsam beginnenden, milden respiratorischen Symptomen bis hin zu einer eher stürmischen Manifestation einer respiratorischen Insuffizienz $[2,7,13]$. Häufig wird dies begleitet von extrapulmonalen Infekten bzw. Infektionen, was die Diagnostik weiter erschwert. Ist wie bei der angeborenen Zwerchfellhernie bei später Manifestation eine atypische Auskultationsbefundung des Thorax mit dort nachweisbaren Darmgeräuschen auszumachen, ist stets eine umgehende Diagnostik mit Röntgenaufnahme des Thorax erforderlich.

Eine operative Behandlung ist bei rechtzeitiger Indikationsstellung aufgrund der bis zur Manifestation der späten Zwerchfellhernie regelrechten Lungenfunktion in aller Regel erfolgreich, da schwerwiegende Lungenhypoplasien, die die lebenslimitierende Problematik bei der angeborenen Form bedingen, hier fehlen.

Die die oben zitierten Mitteilungen betten sich ein in frühere Artikel aus der Klinischen Pädiatrie, wo die Problematik der Zwerchfellhernie bzw. übergeordneter respiratorischer Versagenszustände bei Neugeborenen adressiert wurden $[10,17]$. 
Tierexperimentell lassen sich die angeborenen Zwerchfellhernien durch einen schwerwiegenden Vitamin-A-Mangel bei den Muttertieren bzw. durch eine pränatale Nitrofen-Applikation an die schwangeren Tiere induzieren [3]. In genetischen Modellen wurden WT1-mutante Mäuse mit der typischen BochdalekHernie beschrieben $[3,11]$.

Derzeitige Untersuchungen zur Pathogenese der Zwerchfellhernie auf der genetischen Ebene bilden eine Brücke zu den syndromal auftretenden Erkrankungen wie Zwerchfellhernie, welche u.a. das Denys-Drash-Syndrom oder das Meacham-Syndrom beinhalten [3].

Eine Koinzidenz eines neonatalen Diabetes und einer angeborenen Zwerchfellhernie wurde jüngst berichtet [18], inwieweit es sich hierbei aber um eine gemeinsame Ätiologie oder ein zufälliges Zusammentreffen einer mittelhäufigen und einer sehr seltenen Erkrankung handelt, muss unklar bleiben.

Die Suche nach Kandidatengenen geht neben den zuvor geschilderten Befunden in zweierlei Richtungen:

Zum Einen wird in etablierten tierexperimentellen Modellen die Suche nach Kandidatengenen fortgesetzt, zum Anderen findet eine Sammlung von DNA-Proben betroffener Kinder mit dem Ziel einer genomweiten Analyse statt $[4,16]$.

Diese Methodik ist naturgemäß bei der Seltenheit der Erkrankung beim Menschen hochgradig aufwendig, weshalb hierbei noch keine systematischen Daten vorliegen. Dagegen ist der Ansatz der Analyse unter experimentellen Bedingungen im murinen Modell fortgeschritten, wo es eine Reihe von 27 Kandidatengenen im experimentellen Zwerchfellhernien-Modell identifiziert werden konnten, die u.a. in die Signaltransduktionskette des Vitamin A (retinoic acid - RA) sowie der Signalwege des Wnt- und des Transforming-Growth-Factor-beta-Gens sowie des Ephrin-Gens zuzuordnen sind [16,19].

Auch diese Betrachtung belegt, dass die Etablierung von Registern der Erkrankung und Biobanken notwendig sind, um weitere Aufschlüsse über die molekulare Pathogenese $\mathrm{zu}$ gewinnen $[9,14]$. Diese Forschungsansätze ermöglichen präventives Vorgehen, um weitere Fortschritte bei dieser schwerwiegenden Erkrankung zu erzielen.

\section{Literatur}

1 Adzick NS, Harrison MR, Glick PL et al. Diaphragmatic hernia in the fetus: prenatal diagnosis and outcome in 94 cases. J Pediatr Surg 1985; 20: 357-361

2 Bode S, Greiner P, Franke $M$ et al. Late presentation of congenital diaphragmatic hernias-report of three cases. Klin Padiatr 2012; 224: 465-466
3 Clugston RD, Klattig J, Englert $C$ et al. Teratogen-induced, dietary and genetic models of congenital diaphragmatic hernia share a common mechanism of pathogenesis. Am J Pathol 2006; 169: 1541-1549

4 Clugston RD, Zhang W, Greer JJ. Gene expression in the developing diaphragm: significance for congenital diaphragmatic hernia. Am J Physiol Lung Cell Mol Physiol 2008; 294: L665-L675

5 Dahlheim $M$, Witsch $M$, Demirakca $S$ et al. Congenital diaphragmatic hernia - results of an ECMO-centre. Klin Padiatr 2003; 215: 213-222

6 Dekoninck P, Gratacos E, Van MT et al. Results of fetal endoscopic tracheal occlusion for congenital diaphragmatic hernia and the set up of the randomized controlled TOTAL trial. Early Hum Dev 2011; 87: 619-624

7 Delaney CA, Rozance PJ, Sandoval JA et al. Late onset diaphragmatic hernia complicated by intestinal strangulation. Curr Opin Pediatr 2012; 24: 274-276

8 Deprest J, De CP. Antenatal management of isolated congenital diaphragmatic hernia today and tomorrow: ongoing collaborative research and development. Journal of Pediatric Surgery Lecture. J Pediatr Surg 2012; 47: 282-290

9 Gobel $U$, Gortner L. Disease management programs for adults with often diseases and competence networks for children and adolescents with rare diseases. Klin Padiatr 2011; 223: 1-3

10 Hofmann SR, Stadler K, Heilmann A et al. Stabilisation of cardiopulmonary function in newborns with congenital diaphragmatic hernia using lung function parameters and hemodynamic management. Klin Padiatr 2012; 224: e1-e10

11 Holder AM, Klaassens $M$, Tibboel D et al. Genetic factors in congenital diaphragmatic hernia. Am J Hum Genet 2007; 80: 825-845

12 Kilian AK, Busing KA, Schuetz EM et al. Fetal MR lung volumetry in congenital diaphragmatic hernia $(\mathrm{CDH})$ : prediction of clinical outcome and the need for extracorporeal membrane oxygenation (ECMO). Klin Padiatr 2009; 221: 295-301

13 Navarro-Crummenauer B, Breitmeier D, Rauh $W$ et al. Death of an Infant due to an Undiagnosed Congenital Diaphragmatic Hernia. Klin Padiatr 2012; 224: 463-464

14 Paul T. The PAN study: the first step into the future. Klin Padiatr 2010; 222: 281

15 Roehr CC, Hansmann G, Hoehn T et al. The 2010 Guidelines on Neonatal Resuscitation (AHA, ERC, ILCOR): similarities and differences - what progress has been made since 2005? Klin Padiatr 2011; 223: 299-307

16 Russell MK, Longoni M, Wells J et al. Congenital diaphragmatic hernia candidate genes derived from embryonic transcriptomes. Proc Natl Acad Sci USA 21 2012; 109: 2978-2983

17 Schaible T, Veit M, Tautz J et al. Serum cytokine levels in neonates with congenital diaphragmatic hernia. Klin Padiatr 2011; 223: 414-418

18 Topiol ES, Minarich LA, Williams CA et al. Neonatal diabetes mellitus and congenital diaphragmatic hernia: coincidence or concurrent etiology? Int J Pediatr Endocrinol 2012; 2012: 21

19 Wat MJ, Veenma D, Hogue J et al. Genomic alterations that contribute to the development of isolated and non-isolated congenital diaphragmatic hernia. J Med Genet 2011; 48: 299-307 